\title{
Infrared Radiation From Interstellar Grains
}

\author{
Wayne A. STEIN \\ Princeton University \\ Princeton, New Jersey
}

DURING THE PAST SEVERAL YEARS there has been a growing interest in the possibility of observing infrared radiation at wavelengths greater than $10 \mu$ from various astronomical objects. For example, Gould in references 1 and 2 has suggested trying to observe several lines in the infrared with emphasis on the $12.8-\mu$ line of $\mathrm{Ne}^{+}$and the $28-\mu$ line of molecular hydrogen. The possibility of being able to observe the emission from interstellar grains in the wavelength region between $10 \mu$ and $100 \mu$ was suggested in a discussion presented in references 3 and 4 . The intensity expected from thermal emission by the grains has also been investigated by F. D. Kahn at Manchester.

The present paper indicates information about interstellar grains that could be gained by measuring the intensity as a function of wavelength and the polarization of radiation emitted by the grains in the far infrared. Because of uncertainties in the knowledge of the radiation field from stars in space and in the composition and temperatures of the grains, the models for the far infrared radiation calculated herein may not agree in detail with the results of the observations. It is the purpose of the present discussion to indicate the kind of measurements that should be made in order to gain better knowledge of these quantities.

\section{EXPECTED INTENSITY}

The total radiation flux incident on the grains is about $5.3 \times 10^{-3}$ $\mathrm{erg} / \mathrm{cm}^{2}$-sec. (See ref. 5.) If one-tenth of this radiation is absorbed by the grains, it must be reemitted in the far infrared. If the infrared radiation is emitted isotropically over 4- $\pi$ steradians, the intensity observed at the Earth would be

$$
\left(\frac{5.3 \times 10^{-4}}{4 \pi}\right) 4 \pi r^{2} n l \text { ergs } / \mathrm{cm}^{2} \text {-sec-sr }
$$

where $r$ is the grain radius, $n$ is the density of grains, and $l$ is the path 
length. For $n=2 \times 10^{-13} / \mathrm{cm}^{3}, r=2 \times 10^{-5} \mathrm{~cm}$, and $l=20 \mathrm{kpc}$, the intensity of radiation in the galactic plane is $2.5 \times 10^{-10} \mathrm{watt} / \mathrm{cm}^{2}-\mathrm{sr}$. This intensity could be detected with modern far infrared detectors.

The most obvious difficulty involved in making these observations is the interfering effects of emission from water vapor in the Earth's atmosphere. The observations could possibly be made from balloon altitudes, but the detectors more likely would have to be flown above the Earth's atmosphere by rockets.

\section{TEMPERATURES AND SPECTRA OF THE GRAINS}

Four extreme types of interstellar grains are considered: metal, graphite, dielectric (ice), and Platt particles. The large molecules proposed by Platt (ref. 6) could immediately be distinguished from the other models of the grains by the presence or absence of far infrared radiation. These large molecules, because of their size, would contribute no radiation in the far infrared.

The equation of energy balance (ref. 7) leads to the temperatures and spectra of radiation from the grains. As a simplified example of the results one might expect, consider the spectral distribution for a dilution factor of $10^{-14}$ and an absorption efficiency of $1 / 10$. The temperatures of grains of radius $0.2 \mu$ would be $74^{\circ} \mathrm{K}$ for a good conductor, $30^{\circ} \mathrm{K}$ for graphite, and $15^{\circ} \mathrm{K}$ for ice under these conditions. The wavelengths of maximum emission would then be $28 \mu$ for a good conductor, $70 \mu$ for graphite, and $125 \mu$ for ice, as shown in figure 1 .

The spread of these spectra due to variations in dilution factor $W$ and radius of particles can be shown for metal particles by the result

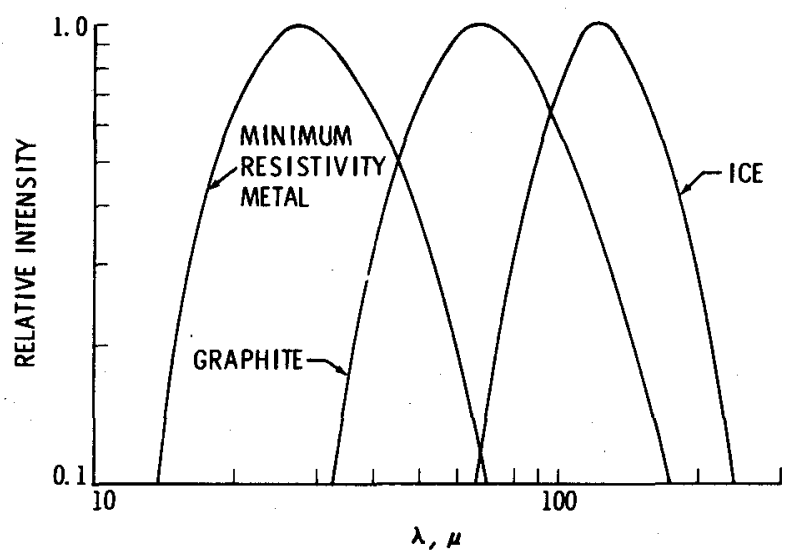

FIGURE 1.-Expected spectra of emission from interstellar grains of various compositions normalized to $l$ at the wavelength of maximum intensity. 
of the equation of energy balance in which the particle temperature $T_{p}$ varies as $(W / r)^{1 / 6}$ or slower. A change by a factor of 100 in $W$ would then result in a change by a factor of 2.15 in temperature. A similar result for dirty ice grains is presented in reference 8 . For ice, a change by a factor of 100 in dilution factor resulted in a change by a factor of from 2 to 2.5 in temperature. The extreme examples of a good conductor and ice are separated by a factor of 5 in temperature. Thus it seems possible that under some circumstances, low temperature, high conductivity grains might be difficult to distinguish from high conductivity, low temperature grains, but extreme fluctuations in $W$ or $r$ would be necessary to lose the contrast between the spectra of high conductivity grains and ice. At least the temperature of the grains would be determined. Further calculations of details of spectra for composite grains and other complications should probably await observation of the radiation.

\section{POLARIZATION}

If the true source of the polarization of starlight at visible wavelengths is absorption of light by alined, elongated, interstellar grains, then the far infrared radiation from the grains should also be polarized. In order to compare the percentage of polarization from the two mechanisms, only the case of perfectly alined grains was considered.

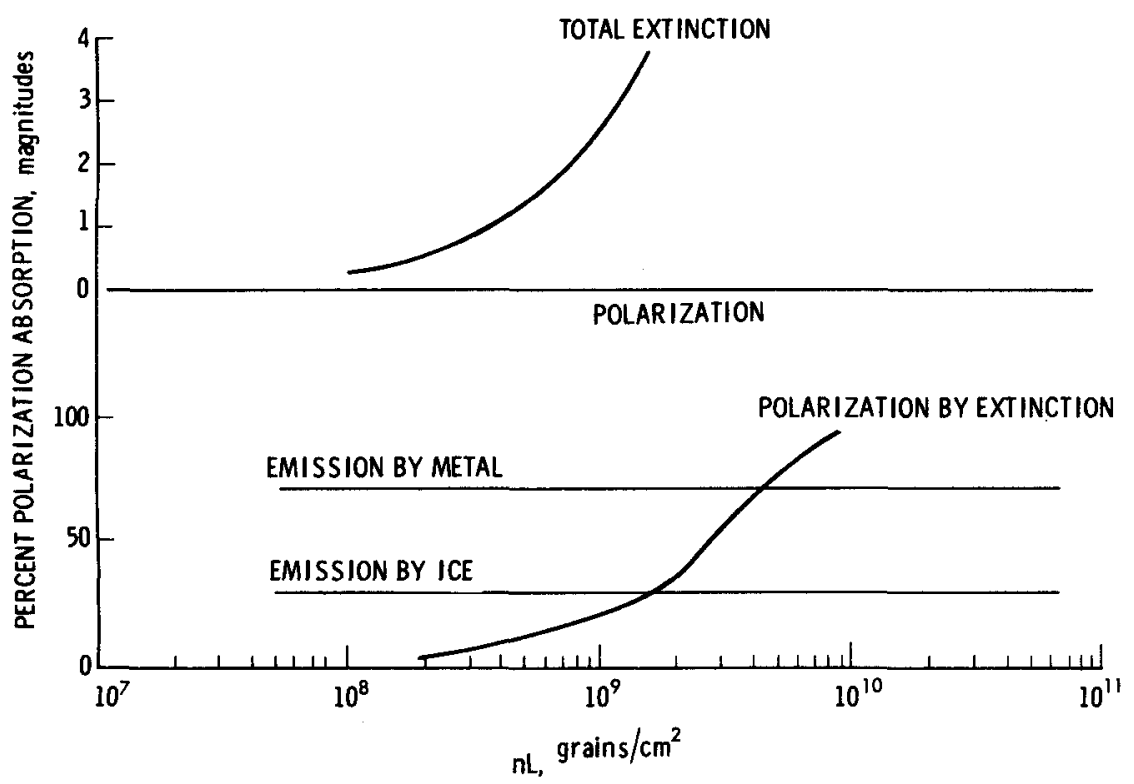

FIGURE 2,- Total extinction and polarization at visible wavelengths due to extinction by grains compared with the polarization of emitted infrared as a function of the number of grains per square centimeter in the line of sight for grains with ratio of axes of 2 . 
Figure 2 shows an example of the total extinction and polarization of starlight at visible wavelengths as a function of the number of grains per square centimeter in the line of sight for grains with a ratio of axes of 2. The polarization of the emitted far infrared as calculated for ice and metal grains is shown for comparison. It can be seen that the polarization of emitted infrared radiation from the grains for this example would be larger than the polarization of starlight at visible wavelengths under practically all conditions. In addition, it should be pointed out that since the absorption of far infrared radiation is small, observations of polarization could be made to large distances from the- Earth. Thus the far infrared distribution of energy with wavelength and the polarization of radiation from interstellar grains would yield new information in the study of the grains.

\section{REFERENCES}

1. Gould, R. J.: Infrared Emission Lines from H II Regions. Astrophys. J., vol. 138, 1963, p. 1308.

2. Gould, R. J.: The Contraction of Molecular Hydrogen Protostars. Astrophys. J., vol. 140, 1964, p. 638.

3. Spitzer, L.: Proceedings of Third Symposium on Cosmical Gas Dynamics. Part VIII: Summaries and Conclusions. Rev. Mod. Phys., vol. 30, 1958, p. 1107.

4. Drake, F.: Proceedings of Third Symposium on Cosmical Gas Dynamics. Part VIII: Summaries and Conclusions. Rev. Mod. Phys., vol. 30, 1958, p. 1107.

5. Allen, C. W.: Astrophysical Quantities, Univ. of London, The Athlone Press, 1963.

6. Platt, J. R.: On the Optical Properties of Interstellar Dust. Astrophys. J., vol. 123, 1956, p. 486.

7. Van de Hulst, H. C.: Optics of Spherical Particles. Rech. Astron. Obs. Utrecht, vol. 11, pt. 1, 1946.

8. Greenberg, J. M.: Interstellar Grains. Ann. Rev. Astron. Astrophys. vol. 1, 1963, p. 267.

\section{DISCUSSION}

Field: It occurs to me that one expects the distribution of the infrared to mimic that of reflection nebulae because reflection nebulae represent combinations of dust concentration and stellar radiation which give detectable scattered light. The same combination gives detectable infrared emission; therefore, such emission will probably be very patchy, as is the scattered light. The surface brightness may well be much higher than the average value you have computed for certain regions often associated with reflection nebulae.

Stein: Yes, I agree. One might be able to see individual clouds, depending upon how the numbers of particle density and path length are estimated. The wavelength of emission in particular regions might occur at shorter wavelengths also.

Field: The point is that it may not be the diffuse light you want to look for, but rather those patches which are quite near the stars; their angular dimensions are very much smaller. 
Stein: Yes. But then also one would look at shorter wavelengths than the $100-\mu$ region.

Donn: Do you have an estimate for the background light from the stars in the far infrared?

Stein: I don't know anything about stars at $100 \mu$. I have made estimates of the radiation at these wavelengths by extrapolating radio spectra of radio sources. I think one might be able to see the Sag A radio source. I think that the emission from the Zodiacal light would occur at shorter wavelengths, somewhere in the $1-\mu$ to $10-\mu$ range.

Hall: Have you made any estimates of how many watts per steradian would be received from these three types of grains at the Earth's surface after passing through the water vapor in the atmosphere?

Stein: It's hopeless.

Hall: Even at the shorter wavelengths? There is a window at $8 \mu$ to $14 \mu$ and one at $22 \mu$ to $24 \mu$.

Stein: $\mathrm{Oh}$, but the latter window is very small. One needs a very wide band in order to collect a lot of radiation. The problem isn't so much the absorption, but the fact that the water vapor in the Earth's atmosphere absorbs radiation from the Earth and then reemits; it is that flux of emission from the Earth's atmosphere that would cause the problem.

Hall: For the background?

Stein: That's right. This is what makes me very dubious about whether it can be done from balloons or not. One might have to use rockets to get above the water vapor.

W. E. Thompson: Is there any problem of infrared radiation from the instrument itself in the far infrared?

Stein: This involves chopping techniques and cooling of components. When one observes far infrared radiation like this, one chops the region he is observing against the background. The direct-current component is no problem; the problem involves cooling of the detectors and other components. 